\title{
Development of a Terawatt Class Sub-10 fs Laser System
}

\author{
Abdolreza AMANI EILANLOU, ${ }^{1,2,3}$ Yasuo NABEKAWA, ${ }^{1}$ Kenichi L. ISHIKAWA, ${ }^{2,3}$ Hiroyuki TAKAHASHI, ${ }^{2}$ and \\ Katsumi MIDORIKAWA ${ }^{1}$ \\ ${ }^{1}$ RIKEN, 2-1 Hirosawa, Wako-shi, Saitama 351-0198 \\ ${ }^{2}$ Univ. of Tokyo, 7-3-1 Hongo, Bunkyo-ku, Tokyo 113-8656 \\ ${ }^{3}$ JST, 4-1-8 Honcho, Kawaguchi-shi, Saitama 332-0012
}

(Received January 28, 2008)

\begin{abstract}
We have developed an amplifier chain of Ti:Sapphire laser with a spectral width of more than $200 \mathrm{~nm}$, potentially generates a terawatt sub-10 fs pulse. This laser system consists of a Ti:Sapphire oscillator followed by a pulse stretcher, dispersion compensator with an SLM and the amplifier chain. The first amplifier is a regenerative one pumped by a green laser with energy of $11.5 \mathrm{~mJ}$ at $1 \mathrm{kHz}$. We could successfully compensate for the gain narrowing during regenerative amplification by using high-damage-threshold chirped mirrors and specially designed partial mirrors in the cavity, resulting in pulse energy of $73 \mu \mathrm{J}$. The output pulse is sent to a beam-slicer to reduce the repetition rate to $10 \mathrm{~Hz}$. After 5-pass amplification in a multi-pass amplifier pumped by the second harmonic of a YAG laser with an energy of $180 \mathrm{~mJ}$, we obtained an amplified pulse energy of $30 \mathrm{~mJ}$.
\end{abstract}

Key Words: High-intensity ultrafast lasers, Ti:Sapphire lasers, Sub-10 fs, Terawatt

\section{Introduction}

The intense few-cycle laser pulses are useful for a variety of applications such as single attosecond pulse generation with a high-order harmonic field or generation of soft X-rays in the water window region $(2.32 \mathrm{~nm} \sim 4.37 \mathrm{~nm})$. Direct amplification of such short laser pulses in the visible to the near infrared spectral region, without utilizing the nonlinear spectral broadening effect, has been realized by applying state-of-the-art technique of optical parametric chirped pulse amplification (OPCPA) ${ }^{1,2)}$ Although the OPCPA has the advantage of attaining sufficiently broad gain bandwidth for amplifying sub-10 fs pulses with a high single-pass gain, it requires an accurate synchronization of a pumping laser pulse with a duration of less than 100 ps to the signal pulse. The technical difficulty for developing such a customized pumping laser system seems a significant issue for the widespread use of OPCPA laser systems in scientific communities.

The chirped pulse amplification (CPA) system of Ti:Sapphire laser, on the other hand, can be pumped by commercially available Q-switched laser sources and is a conventional tool in many scientific fields. Yet, it has been difficult to amplify sub-10 fs pulses directly in a terawatt class CPA system owing to the fact that spectral width could be easily restricted by gain narrowing of the laser medium itself and the narrow bandwidth of high-damage-threshold (HDT) mirrors used in the amplifiers. Thus, the final goal of our research is to develop a terawatt class sub-10 fs laser system, applying Ti:Sapphire crystal as the amplification medium pumped by the commercially available high power Q-switched green lasers. In this paper, we report on the characteristics of the specially designed optical elements and the development of our laser system, which potentially generates terawatt class sub-10 fs pulses directly from a Ti:Sapphire CPA system.

\section{The sub-10 fs regime}

The major problems in the amplification of sub-10 fs pulses are: (i) gain narrowing which is spectral narrowing originating from the gain profile of a Ti:Sapphire laser, (ii) the limit of the spectral width of HDT mirrors, and (iii) pulse broadening originating from the high-order dispersion due to the optical elements used in the laser system. ${ }^{3)}$

To compensate for the gain narrowing in the regenerative amplifier of our CPA system, we developed several specially designed partial mirrors to give a loss to the amplifier at the high gain region of the Ti:Sapphire laser. We used an iterative algorithm to design the coating of these partial mirrors so that it had a transmission curve close to the inverted gain profile of Ti:Sapphire crystal near the central wavelength of $800 \mathrm{~nm}$. The calculated transmission and group delay dispersion (GDD) of these partial mirrors appear in Fig. 1. They have a low GDD which makes it possible to be used in the cavity of a regenerative amplifier for regenerative pulse shaping and obtaining broadband amplified spectra. ${ }^{3,4)}$

We investigated how these partial mirrors are effective for compensating the gain narrowing by inserting them in a test

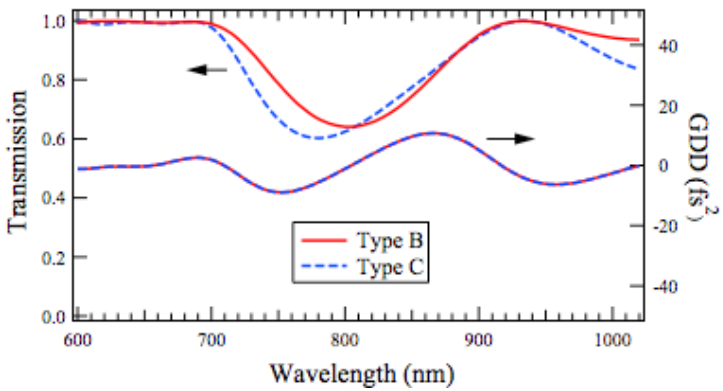

Fig. 1 Calculated transmission and GDD of the partial mirrors. Type $\mathrm{C}$ (the dashed line) is designed to have a valley in its transmission curve at a shorter wavelength compared to that for Type B (the solid line). 


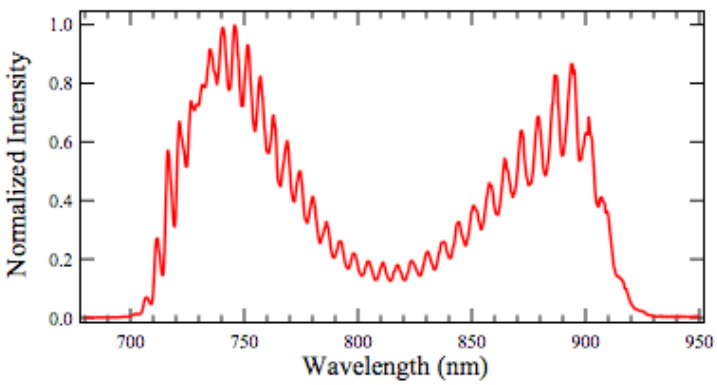

Fig. 2 Output spectrum of the Q-switched oscillation of a test cavity pumped by a high-energy Q-switched green laser.

cavity. The Ti:Sapphire crystal in the test cavity was pumped by a Q-switched green laser at a repetition rate of $1 \mathrm{kHz}$. To avoid spectral limit due to reflection, we used four Pellin-Broca prisms to build this test cavity. ${ }^{5)}$ They reflect the pulse by total reflection and have a low loss, since they are used at Brewster angle incidence. Figure 2 shows the output spectrum of the Q-switched oscillation of the test cavity. The spectral width was as broad as approximately $240 \mathrm{~nm}$ on foot, so that pulse duration of less than $10 \mathrm{fs}$ at the Fourier limit could be expected when these partial mirrors are applied to the regenerative amplifier.

In order to solve the second issue which is the limit of the spectral width of HDT mirrors, we developed several broadband HDT mirrors and confirmed that the total dispersion of the laser system would be compensable by measuring their spectral dispersion with a spectral interferometry technique using the broadband spectrum of a Ti:Sapphire laser oscillator. Figure 3 shows the calculated reflectivity and the measured GDD of these chirped mirrors. To compensate for their GDD oscillation they are used in pairs.

To make sure that the measurement technique is precise, we also measured the GDD of these chirped mirrors in pairs and compared the result with their sum of GDD. Figure 4 shows the details of this measurement. As a result, their GDD oscillation is low enough to be compensated by a spatial light modulator and has a smooth curve.

Reaching a peak power of more than one terawatt and pulse duration of less than 10 fs from only a regenerative amplifier is a rather difficult challenge. Instead, amplifying the output pulse from the regenerative amplifier in a power amplifier

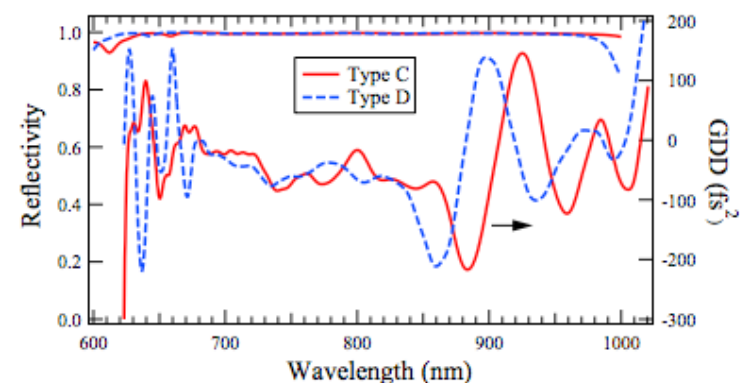

Fig. 3 Calculated reflectivity (the upper curves) and the measured GDD of the broadband HDT chirped mirrors to be used in a regenerative amplifier.

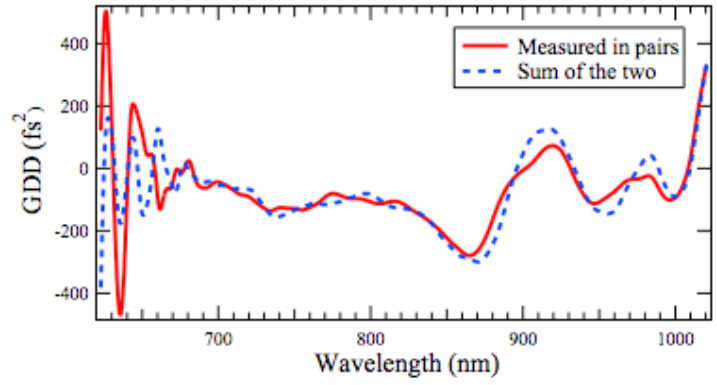

Fig. 4 Measured GDD of the chirped mirrors in pairs (the solid line), compared with the sum of the measured GDD of the two mirrors.

such as a bow-tie multi-pass amplifier is a better option. Thus, we designed an S-polarized $45^{\circ}$ broadband HDT mirror to be used in a multi-pass amplifier. Such a laser mirror should also have a low high-order dispersion so that the dispersion of the laser system remains compensable. To confirm that it has a low dispersion, we measured its GDD and obtained a result quite close to its calculated GDD value. The result is shown in Fig. 5.

Finally, to compensate for residual dispersion after compressing the pulses in a terawatt class Ti:Sapphire CPA system, we calibrated a spatial light modulator (SLM) with the ultra-broadband spectrum of a Ti:Sapphire laser oscillator and made it ready to be used in the laser system. The SLM is supposed to compensate for the GDD oscillation of the chirped mirrors as well as the high-order dispersion of the laser system.

\section{Development of the laser system}

Based on the results of these preliminary experiments, we started developing an amplifier chain of Ti:Sapphire laser with a spectral width of more than $200 \mathrm{~nm}$, which potentially generates a terawatt sub-10 fs pulse. This laser system consists of a commercial Ti:Sapphire oscillator having a pulse duration of 7 fs. The oscillator is followed by an Öffner type pulse stretcher consisting of a $600 \mathrm{~g} / \mathrm{mm}$ diffraction grating and a telescope with a radius of curvature of $-600 \mathrm{~mm}$ for the concave mirror and $300 \mathrm{~mm}$ for the convex mirror. By such a configuration, the input pulse is stretched to 200 ps. The stretcher is followed by a dispersion compensator with an SLM having 640 channels. To avoid the loss due to the use of

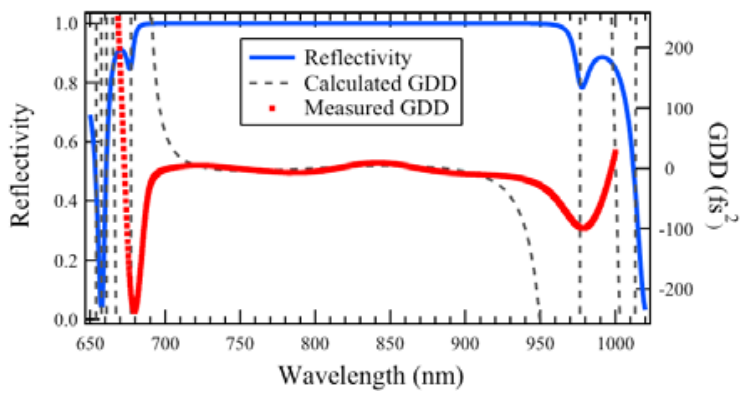

Fig. 5 Calculated reflectivity (the upper blue solid line) and GDD (the dashed line) and the measured GDD (the red dots) of the broadband HDT $45^{\circ}$ laser mirrors to be used in a multi-pass amplifier. 


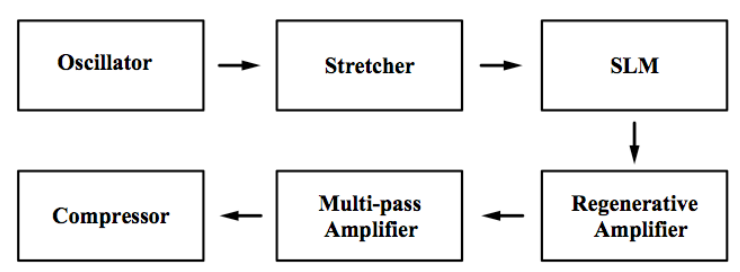

Fig. 6 Schematic of the laser system.

diffraction grating pairs in the SLM 4f-setup, we used a sequence of three Brewster prisms to provide enough angular dispersion. The output pulse of the SLM 4f-setup is injected into the amplifier chain followed by a diffraction grating based pulse compressor. The schematic of the laser system including the amplifier chain is shown in Fig. 6.,6)

The first stage of the amplifier chain is a regenerative one pumped by a Q-switched green laser with pulse energy of 11.5 $\mathrm{mJ}$ at a $1 \mathrm{kHz}$ repetition rate. It consists of two concave chirped mirrors with a radius of curvature of $-600 \mathrm{~mm}$ and two other flat chirped mirrors making an X-fold cavity. The smooth GDD of these broadband HDT chirped mirrors appears in Fig. 3 and Fig. 4. We could successfully compensate for the spectral and gain narrowing during the regenerative amplification by using the broadband HDT chirped mirrors and the specially designed partial mirrors (Type B and Type C in Fig. 1) in the cavity. The obtained pulse energy and spectral width were $73 \mu \mathrm{J}$ and $240 \mathrm{~nm}$, respectively. The spectrum of this regenerative amplifier appears in Fig. 7. It is a broadband spectrum and the magnified spectrum shown in the inset of Fig. 7 reveals that a reasonable amount of amplified photons exist in the rather low intensity region of $750 \mathrm{~nm}$ to $850 \mathrm{~nm}$.

The output pulse from the regenerative amplifier is sent to a beam-slicer to isolate the two stages in the amplifier chain and to reduce the repetition rate to $10 \mathrm{~Hz}$. Also it reduces the ASE from the regenerative amplifier. After 5-pass amplification in the second stage of the amplifier chain which is a bowtie multi-pass amplifier pumped by the second harmonic of a Q-switched YAG laser with a pulse energy of $180 \mathrm{~mJ}$, we obtained an amplified pulse with a pulse energy of $30 \mathrm{~mJ}$ and a spectral width sufficiently broad to form a sub-10 fs pulse. The amplified spectrum is shown in Fig. 8. Although it has an

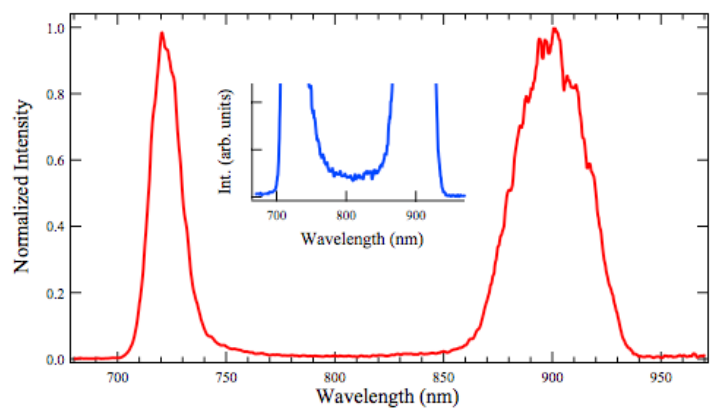

Fig. 7 Amplified spectrum from the regenerative amplifier. Inset shows the magnified spectrum

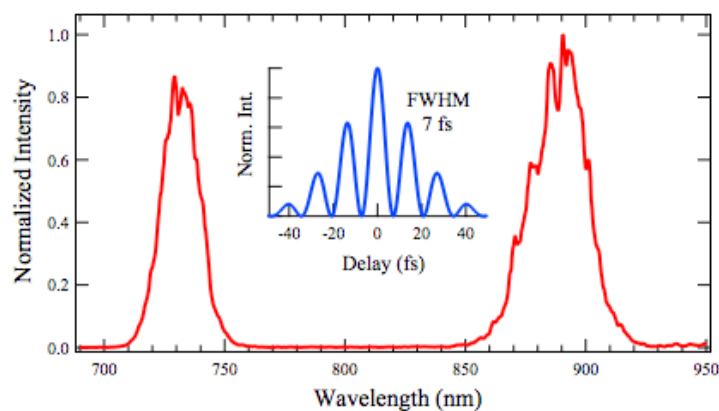

Fig. 8 Amplified spectrum from the multi-pass amplifier. Inset shows the calculated transform-limited temporal profile of this spectrum to show that it has a broad bandwidth to support the generation of sub-10 fs pulses.

acceptable shape in the short wavelength and the long wavelength sides, still it does not have any components in the range of $750 \mathrm{~nm}$ to $850 \mathrm{~nm}$. This problem must be solved first to reach our final goal.

\section{Conclusions}

We solved the major problems in amplification of sub-10 fs pulses. The measured GDD of the broadband high-damage-threshold mirrors used in the regenerative amplifier and those used in the multi-pass amplifier ensure that the total dispersion of the laser system is compensable by application of an SLM, which was calibrated for this laser system. The partial mirrors as well showed to be a prominent way of gain narrowing dispersion in the sub-10 fs regime.

We also developed an amplifier chain of Ti:Sapphire laser with a spectral width of more than $200 \mathrm{~nm}$, which potentially generates a terawatt sub-10 fs pulse. The amplified spectrum does not have any components in the range of $750 \mathrm{~nm}$ to 850 $\mathrm{nm}$, which has a root in the amplified spectrum from the regenerative amplifier. Therefore, we plan to improve the spectral shape of the amplified pulse through modifications of the specially designed partial mirrors in the cavity of the regenerative amplifier. Then using the grating based pulse compressor and the calibrated SLM, we will compensate for the dispersion to generate a transform-limited pulse and accomplish the laser system for generating terawatt sub-10 fs laser pulses in the near future.

\section{References}

1) S. Witte, R. TH. Zinkstok, A. L. Wolf, W. Hogervorst, W. Ubachs and K. S. E. Eikema: Opt. Express 14 (2006) 8168.

2) F. Tavella, A. Marcinkevicus and F. Krausz: Opt. Express 14 (2006) 12822.

3) H. Takada, M. Kakehata and K. Torizuka: Opt. Lett. 31 (2006) 1145.

4) C. P. J. Barty, G. Korn, F. Raksi, C. Rose-Petruck, J. Squier, A. C. Tien, K. R. Wilson, V. V. Yakolev and K. Yamakawa: Opt. Lett. 21 (1996) 219.

5) Y. Nabekawa, Y. Shimizu and K. Midorikawa: Opt. Lett. 27 (2002) 1265.

6) H. Takada and K. Torizuka: IEEE J. Sel. Top. Quantum Electron. 12, (2006) 201. 